\title{
Perspectives in Atopic Dermatitis-Optimizing Outcomes: Introduction
}

\begin{abstract}
$A$ topic dermatitis (AD) has a substantial impact on both

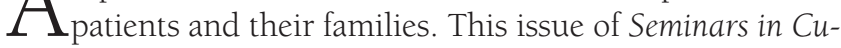
taneous Medicine and Surgery highlights newly recognized aspects of $A D$ pathogenesis as well as the evolution in therapy that offers opportunities for improved outcomes. The faculty members convened for this supplement provide up-to-date evidence that supports some long-held concepts; they also review other recently published data that challenge clinicians to reconsider long-standing ideas about the pathogenesis and treatment of $\mathrm{AD}$. The major topics concerning $\mathrm{AD}$ that are addressed in this supplement include epidemiology, pathogenesis, microbial colonization and infection, comorbidities, disease in adults, prevention and treatment strategies, and improving the patientclinician/parent-clinician partnership in AD management.
\end{abstract}

\section{Epidemiology and Pathogenesis}

The prevalence of $\mathrm{AD}$ has been increasing worldwide over the past several decades, with a current reported prevalence of $10 \%$ to $20 \%$ among children in industrialized countries. ${ }^{1}$ As the authors discuss in the article by Eichenfield et al, ${ }^{2}$ relatively new data from the United States show a prevalence of $\mathrm{AD}$ of $8.7 \%$ to $18 \%$, with interesting prevalence differences from state to state-higher rates are found in Idaho, Nevada, Utah, and the East coastal states. ${ }^{1}$

In the past, discussions of $\mathrm{AD}$ pathogenesis focused on immune dysregulation. Now, however, recent research ad-

Publication of this CME article was jointly sponsored by the University of Louisville Continuing Health Sciences Education and Global Academy for Medical Education, LLC in affiliation with Skin Disease Education Foundation and is supported by an educational grant from Valeant Pharmaceuticals North America Inc.

Dr. Eichenfield has served as a consultant for Anacor, Bayer, and Onset Therapeutics and as a speaker and consultant for Valeant. He has also been an investigator and consultant for Galderma and LEO Pharma as well as an investigator for Amgen, Astellas Pharma US, and Stiefel, A GSK Company.

Lawrence F. Eichenfield, MD, has received an honorarium from Global Academy for Medical Education for his participation in this activity. He acknowledges the editorial assistance of Joanne Still, medical writer, and Global Academy for Medical Education in the development of this continuing medical education journal article. Joanne Still has no relevant financial relationships with any commercial interests. vances have led to an understanding that sets of mutations in skin cell genes are associated with skin barrier defects in AD. Data discussed by Eichenfield et $\mathrm{al}^{2}$ demonstrate that mutations in these genes-especially in the filaggrin gene-are strongly predictive of AD as well as immunoglobulin E sensitization and allergy. These findings have challenged us to consider sensitization and allergy not as primary causes of $\mathrm{AD}$, but as secondary consequences of skin barrier dysfunction. This newer information about pathogenesis is changing approaches to the care of patients with $\mathrm{AD}$.

\section{Colonization and Infection in AD}

It has long been known that Staphylococcus aureus is an important organism in $\mathrm{AD}$, being associated with high rates of colonization and infection and being responsible for impetiginized dermatitis, pustules, and, occasionally, more significant skin, bone, or systemic infections in patients with $\mathrm{AD}$. The paper by Eichenfield et $\mathrm{al}^{2}$ discusses perspectives on the bacterial and viral complications in $\mathrm{AD}$, as well as some of the intriguing data on how normal skin flora-such as Staphylococcus epidermidiscontributes to innate immune reactions. This raises the question whether changes in usual commensal microbes on the skin in patients with AD may affect the cutaneous innate immune system, as well as how $S$. aureus colonization develops and influences the course of $\mathrm{AD}$ over time.

As Paller et $\mathrm{al}^{3}$ note, studies have shown that twice-weekly bathing with sodium hypochlorite solution ("bleach baths") can markedly improve AD by decreasing microbial colonization and reducing the risk for infection. Increasingly, clinical experience also supports the benefit of bleach baths.

\section{Comorbidities in AD}

Simpson and colleagues ${ }^{4}$ highlight the very important emerging information on comorbidities in AD. Although it has long been recognized that $\mathrm{AD}$ is associated with the development of asthma, allergic rhinitis, and food allergy, evidence regarding behavioral, emotional, and psychological comorbidities has been increasingly reported. Particularly interesting are the data 
demonstrating that children with AD may have increased rates of attention-deficit/hyperactivity disorder (ADHD). Autism also may be associated with $\mathrm{AD}$. It is unknown whether $\mathrm{AD}$ is a causal factor in ADHD or other psychological effects, but the data appear to be very strong that there is a significant association. This emerging evidence should strengthen clinicians' conviction to optimize treatment of $\mathrm{AD}$, minimizing its impact on pruritus, skin inflammation, and sleep disturbance, all of which may be contributing to these secondary disabilities.

\section{Adult AD}

Although AD in adults is not uncommon, a paucity of studies has been devoted to this topic, particularly adult-onset AD. Adult-onset AD is discussed in the paper contributed by Ellis and colleagues, ${ }^{5}$ who address the clinical presentation of $\mathrm{AD}$ beginning in adulthood, as well as some of the immunologic differences that are found in these patients. The paper also highlights infectious comorbidities in adult $\mathrm{AD}$ and the significant psychiatric and psychological effects of $\mathrm{AD}$ in this population. Depression, suicidal ideation, a substantial impact on quality of life, choice of occupation, and psychosexual issues are all reported with adult AD.

Considerations for treatment of adults with severe AD that does not respond to topical therapy can include phototherapy and systemic immunosuppressive agents, but we look forward to more-specific, biologic-based strategies in the future.

\section{AD Prevention and Treatment Strategies}

The article by Simpson et $\mathrm{al}^{4}$ highlights strategies for AD prevention. We await future publication of the results of the work of investigators who are studying early-intervention skin care targeting abnormal skin barrier function to determine whether this can minimize the development or improve the course of $\mathrm{AD}$.

To optimize AD outcomes, a broad knowledge of skin management strategies and regimens is required, including bathing, nonprescription topical agents, and prescription topical and systemic agents. The article by Paller et $\mathrm{al}^{3}$ discusses strategies for optimizing available therapeutic options for successful treatment of AD.

Bathing and appropriate use of emollients and moisturizers, including targeted barrier-repair products, can be useful as part of the mainstay of therapy for AD. Anti-inflammatory medications, including topical corticosteroids and topical calcineurin inhibitors (TCIs), can be crucial in AD care. New information on the relative safety of the TCIs has emerged, including long-term registry data and multiple epidemiologic studies.

Many individuals affected with AD may have persistent or frequently flaring eczema. The concept of rotational therapy, or intermittent treatment to minimize flares of the disease, has been very helpful in management. Rotational therapy may involve the use of topical corticosteroids, with switching to a TCI, or relatively long-term intermittent application of corticosteroids or TCIs.
A subset of patients with $\mathrm{AD}$ is insufficiently controlled even with excellent topical therapy. The approach to severe refractory $\mathrm{AD}$ can include hospitalization to remove the patient from the environment and ensure adherence with therapy, or phototherapy, or systemic immunosuppressive therapy.

\section{The Patient/Parent-Clinician Partnership}

The art of achieving high-quality, optimally effective AD care involves working with patients and/or their family to understand their disease and to feel comfortable with interventions for control of their disease. Commonly, treatment failure or response that is poorer than expected is secondary to poor adherence. Optimal management of AD involves not only choosing the right therapeutic agents but also effectively explaining regimens of care to patients and their families, encouraging appropriate use of medications, dealing with patient concerns or fears about their products, and having appropriate follow-up to assess their therapeutic efficacy.

The article by Mancini et $\mathrm{al}^{6}$ discusses the patient-clinician/parent-clinician partnership in AD management. Unfortunately, there is tremendous evidence showing poor adherence to care of patients with AD. There has been an evolution in AD management, recognizing that appropriate therapeutic education can make a huge impact on the course of a disease. This article also includes a helpful personal-care-plan information page and form; the care plan may be copied by clinicians and distributed to parents and patients free of charge. (This page also will be posted on the Website, www. globalacademycme.com/sdef using an interactive design to permit computer generation of the personalized care plan.)

Lawrence F. Eichenfield, MD

Professor of Clinical Pediatrics and Medicine (Dermatology)
Chief, Pediatric and Adolescent Dermatology
University of California, San Diego School of Medicine
Rady Children's Hospital
San Diego, California

\section{References}

1. Shaw T, Currie GP, Koudelka CW, et al: Eczema prevalence in the United States: Data from the 2003 National Survey of Children's Health. J Invest Dermatol 131:67-73, 2011

2. Eichenfield LF, Ellis CN, Mancini AJ, et al: Atopic dermatitis: Epidemiology and pathogenesis update. Semin Cutan Med Surg 31:S3-S5, 2012 (suppl 3)

3. Paller AS, Simpson EL, Eichenfield LF, et al: Treatment strategies for atopic dermatitis: Optimizing the available therapeutic options. Semin Cutan Med Surg 31:S10-S17, 2012 (suppl 3)

4. Simpson EL, Eichenfield LF, Ellis CN, et al: Current issues in atopic comorbidities and preventing the atopic march. Semin Cutan Med Surg 31:S6-S9, 2012 (suppl 3)

5. Ellis CN, Mancini AJ, Paller AS, et al: Understanding and managing atopic dermatitis in adult patients. Semin Cutan Med Surg 31:S18-S22, 2012 (suppl 3)

6. Mancini AJ, Paller AS, Simpson EL, et al: Improving the patient-clinician and parent-clinician partnership in atopic dermatitis management. Semin Cutan Med Surg 31:S23-S28, 2012 (suppl 3) 